\title{
Structural Investigation of NCM-Cathode-LLZO-Electrolyte Composites as Promising Candidates for All-Solid-State Batteries Using (Cryo) STEM and PED
}

Thomas Demuth $^{1}$, Till Fuchs ${ }^{2}$, Anuj Pokle ${ }^{1}$, Andreas Beyer ${ }^{3}$, Jürgen Janek ${ }^{2}$ and Kerstin Volz ${ }^{3}$

${ }^{1}$ Materials Science Center (WZMW) and Department of Physics, Philipps-Universität Marburg, Hans Meerwein Strasse, 35032 Marburg, Germany, United States, ${ }^{2}$ Institute of Physical Chemistry \& Center for Materials Research, Justus-Liebig-University, Heinrich-Buff-Ring 17, 35392 Giessen, Germany, United States, ${ }^{3}$ Materials Science Centre and Department of Physics, Philipps University Marburg, HansMeerwein-Straße 6, Marburg, 35032, Germany, Germany

All-solid-state batteries (ASBs) are considered to replace conventional lithium-ion batteries as the next generation of energy storage, offering higher energy density, longer cycle life and better safety ${ }^{1}$.

One of the limiting factors prolonging the breakthrough of ASBs is the solid electrode-solid electrolyte interphase impedance ${ }^{2}$. Layered oxides like $\mathrm{LiNi}_{0.5} \mathrm{Co}_{0.2} \mathrm{Mn}_{0.3} \mathrm{O}_{2}$ (NCM 523) are known to suffer from capacity fade during cycling due to the formation of spinel- or rocksalt-like phases at the particles' surface ${ }^{3}$. Moreover, it has been shown for $\mathrm{Li}_{1.2} \mathrm{Ni}_{0.2} \mathrm{Mn}_{0.6} \mathrm{O}_{2}$ that such surface modifications may also occur during cathode synthesis ${ }^{4}$.

This study examines electrode-electrolyte composite samples consisting of NCM 662 and Al-doped cubic $\mathrm{Li}_{6.25} \mathrm{Al}_{0.25} \mathrm{La}_{3} \mathrm{Zr}_{2} \mathrm{O}_{12}$ (LLZO) in respect of the influence of the sintering temperature on the formation of surface modification layers using multiple transmission electron microscopy techniques.

For this purpose, LLZO and NCM composites sintered at $500^{\circ} \mathrm{C}$ and $600^{\circ} \mathrm{C}$, as well as a sample that is only isostatically pressed but not sintered, are prepared using a focused ion beam approach. As the LLZO and NCM secondary particles are loosely attached, a more elaborate preparation procedure is undertaken in order to prevent the lamella from breaking apart during the thinning process. Therefore, we cover the lamellae with an all-around deposition layer to create a framework that supports the particles during the subsequent thinning steps.

The atomic structure of the samples is investigated by (high angle) annular darkfield (HAADF) scanning transmission electron microscopy (STEM) as well as brightfield transmission electron microscopy (TEM). Due to the high electron sensitivity of LLZO, low beam dose techniques incorporating cryo STEM are needed to image LLZO particles without inducing irradiation damages to the sample.

Another analysis technique we apply to our samples is precession electron diffraction (PED). With PED an area of the sample is scanned and diffraction patterns for each scan point recorded. Due to the precession of the electron beam, the diffraction patterns are quasi kinematical in nature, which is advantageous for phase determination. This allows us to determine the phases of the primary grains, especially at the NCM-LLZO interface, on a micron-scale.

We report that most NCM primary grains, especially in the non-sintered and sintered at $500^{\circ} \mathrm{C}$ sample, consist predominantly of the layered phase (Figure 1 a).

A phase transition from the layered phase in the bulk to a spinel-like phase at the grains' surface along the lithium diffusion channels can be observed (Figure $1 \mathrm{~b}$ ).

At the interface between LLZO and NCM the NCM particles seem to decompose as multiple phases other than the bulk's layered phase can be observed (Figure $1 \mathrm{c}$ ).

In the sample sintered at $600^{\circ} \mathrm{C}$ we find a higher occurrence of the rocksalt-like phase than in the other two samples (Figure 2).

The combination of the highly precise and local analysis by STEM as well as PED enables us to obtain phase information at a high spatial resolution and over a large area, respectively. 

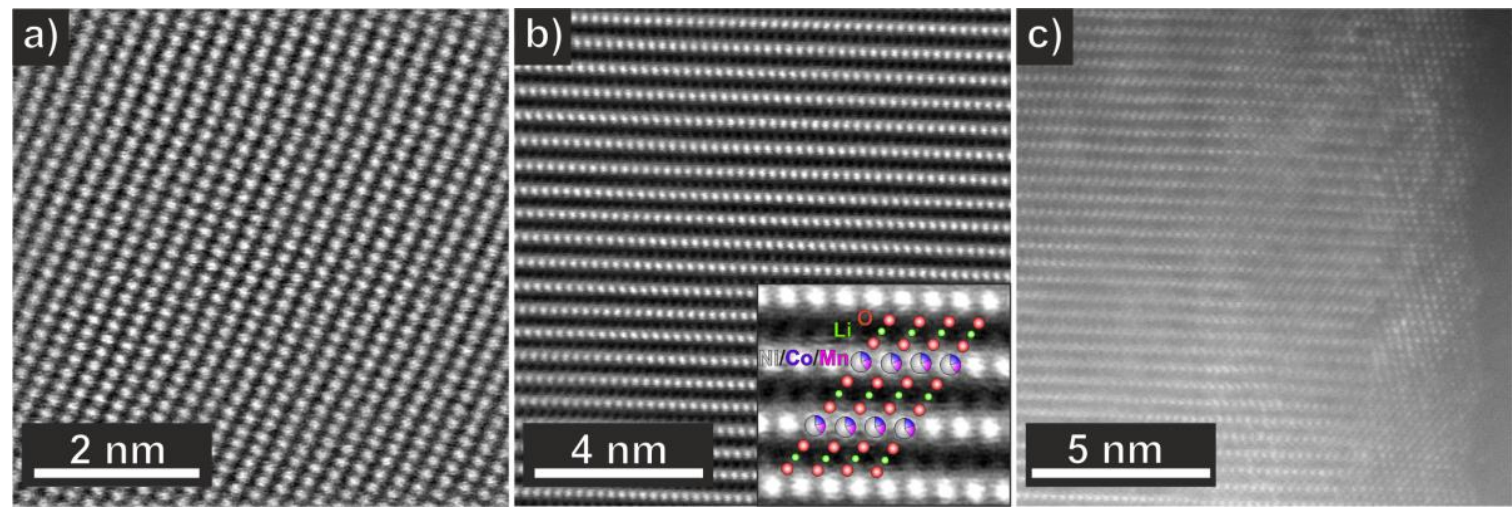

Figure 1. High-resolution HAADF STEM images of an NCM primary grain (sintered at $500^{\circ} \mathrm{C}$ ) showing a) layered structure in the [100] orientation and b) the phase transition from the layered [100] structure in the bulk to a spinel-like structure at the surface. c) TEM Brightfield image of the LLZO NCM interface exhibiting a different phase (blue) at the interface compared to the NCM bulk's layered phase (red).

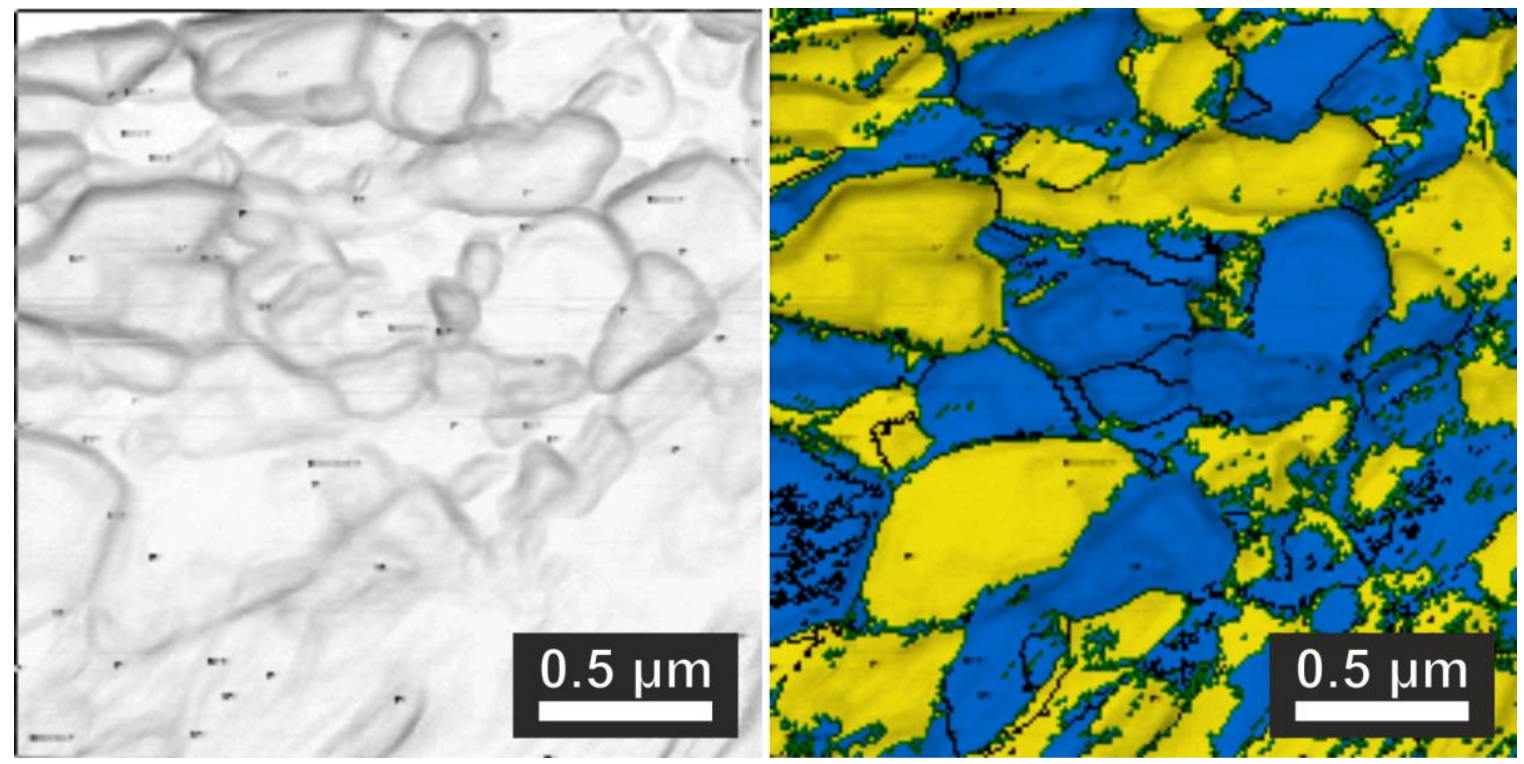

Figure 2. Coefficient Correlation Map a) overlain by a Phase Map b) generated from a PED dataset taken of a NCM secondary particle sintered at $600^{\circ} \mathrm{C}$. To create the Phase Map, the recorded diffraction pattern were matched with computed diffraction pattern of the NCM layered phase (blue) and NiO rocksalt-like phase (yellow). Grain boundaries are highlighted with black lines.

\section{References}

1. Nanda, J., Wang, C., \& Liu, P. Frontiers of solid-state batteries. MRS Bulletin 2018 43(10), 740-745. 2. Wang, Z., et al. In Situ STEM-EELS Observation of Nanoscale Interfacial Phenomena in All-SolidState Batteries. Nano Letters 201616 (6), 3760-3767.

3. Jung, S.-K., et al. Understanding the Degradation Mechanisms of $\mathrm{LiNi}_{0.5} \mathrm{Co}_{0.2} \mathrm{Mn}_{0.3} \mathrm{O}_{2}$ Cathode Material in Lithium Ion Batteries. Adv. Energy Mater. 2014, 4, 1300787

4. Gu, M., et. al. Conflicting Roles of Nickel in Controlling Cathode Performance in Lithium Ion Batteries. Nano Letters 2012 12, 5186-5191. 